\title{
The Influence of Interference Networks in QoS Parameters in a WLAN 802.11g Environment
}

\author{
Jasmine P. L. Araújo ${ }^{1}$, Josiane C. Rodrigues ${ }^{1}$, Simone G. C. Fraiha ${ }^{1}$, Felipe M. \\ Lamarão $^{1}$, Nandamudi L. Vijaykumar ${ }^{2}$, Gervásio P. S. Cavalcante ${ }^{1}$ and \\ Carlos R. L. Francês ${ }^{1}$ \\ 1 Electrical Engineering Faculty, Federal University of Pará (UFPA), 66.075-900, \\ Belém, PA, Brazil \\ \{jasmine, josi,fraiha, gervasio,rfrances\}@ufpa.br, \\ 2 National Institute for Space Research (INPE), Computing and Applied \\ Mathematics Laboratory (LAC), P.O. Box 515, 12245-970, \\ São José dos Campos, SP, Brazil \\ vijay@lac.inpe.br
}

\begin{abstract}
This paper proposes a strategy to determine how much a given network can affect the QoS parameters of another, by interference. In order to achieve this, a measurement campaign was carried out in two stages: firstly with a single AP and later with two APs separated by a distance less than three meters, using the same channel. After the measurement, an analysis of the results and a set of inferences were made by using Bayesian Networks, whose inputs were the experimental data, i.e. QoS metrics such as: throughput, jitter, packet loss, PMOS and physical metrics like power and distance.
\end{abstract}

\section{Introduction}

Wireless Local Area Networks (WLAN) IEEE802.11 have been widely used in the recent years due to their mobility and for being practical in configuring them. They have been a very practical and important solution to industries, companies and historical constructions due to their flexibility and low cost benefits during their installation and utilization. These technologies usually support traffic data generated by web browsing and email applications. In the recent past, they have been considered for voice communication especially in offices [1].

VoIP delivers voice packets over the Internet and consequently the costs are drastically reduced when compared with conventional telephone calls (PSTN). However, these applications require that WLAN installed are capable to support very strict QoS specifications for voice transmission. ITU-T (International Telecommunication Union) G.114 defines, for real-time services, a tolerable rate of packet loss is around $1 \%$ to $3 \%$ and the allowed delay is less than $150 \mathrm{~ms}$ but not greater than $400 \mathrm{~ms}$ [2]. Due to the sensitivity of the specifications, VoIP has been chosen generally to be evaluated in field tests. With respect to the physical layer, three phenomena exert influence on radio transmission: collisions, radio link quality and interference. Collisions are well known phenomena where several 
hosts transmit packets to the environment and at the same time it introduces packets with errors that need to be retransmitted. The link quality does not only depend on the noise level but also on the distribution of stations and the topology of the environment (wall positions, furniture density and others). Moreover, the presence of multiple access points may also introduce interference and thus reduce the transmission quality [3]. Such interference lead to receiving packets with errors and at MAC layer, they have to be retransmitted thus affecting the throughput [4]. Added to this fact, the interference may force transmission at lower rates. As already mentioned, the radio environment factors influence the global WLAN performance. But despite this fact very few analyses were conducted to verify this approach.

The main objective of this paper is to use computational intelligence by employing Bayesian Networks to verify, aiming at quantifying and characterizing this influence. This work will study specifically the effect of the interference. This is because in any environment with WLAN, it probably will have to live together with other sources of interferences in the same frequency band, such as, cordless telephones, microwaves ovens and access points using the same channel.

This paper is organized as follows: Section 2 presents some related work; basic concepts on KDD (Knowledge Discovery in Database), Data Mining and Bayesian networks are shown in Section 3, the environment used for obtaining metrics is presented in Section 4 while Section 5 describes the methodology employed in data acquiring; Section 6 presents the results; Section 7 presents the methodology proposal and finally Section 8 concludes the paper.

\section{Related Works}

This section presents some related works that will be used to compare the results obtained. This also contributes significantly to the research described here. A procedure to design WLAN including VoIP is presented in [5]. It discusses a theoretical model with a suggestion to implement an experimental scenario. An analytical model to plan a WLAN with a guaranteed throughput in an indoor environment is presented in [3]. The algorithm presented explores performance evaluation of the network based on a Markov model of the actual WLAN in which collision and link quality are considered. A cost function was defined as the sum of coverage components, interference and QoS where weights for this function were empirically selected. The objective of [3] is not to choose the least number of access points but keeping the interference at low levels while guaranteeing the throughput.

In [6] it is proposed to integrate radio environment features with an analytical model to evaluate indoor WLAN802.11 performance. An improved Markovian model was proposed by considering signal level, environment topology and geometric distribution of the stations. The model was validated with experiments and suggested as a future work to consider probability of the interference.

The VoIP behavior over 802.11 is discussed in [7] under the perspective of the number of connections that a single access point can support. So, tests are 
conducted to verify how many VoIP sessions can the network support without any degradation in the quality. Based on the results, it was decided to use, in a metric collection campaign, just one single call which was repeated during the experiment to ensure that the observed effects do not influence in the nominal bandwidth due to other VoIP sessions. Thus, the interfering network is responsible for the variation of the QoS parameters. Optimization problems are not treated in [7]; in the research described here, the influence issue will be addressed.

The references [3] and [7] cite other references where hypotheses to evaluate a network were more simplified by considering an ideal channel and without loss. Therefore, the paper described here compares also researches developed from the cited references. Another major difference is that the experiments are conducted with and without interfering network. In addition,[3] and [7] use Markovian model to evaluate just the throughput and the measurements are conducted at a later stage in order to validate the model. In case of this paper, Bayesian network is used for data analysis as well as to verify the influence of radio environment in QoS parameters (throughput, jitter, packet loss and PMOS).

This paper does not yet address the problem of optimization, but by using Bayesian networks, it is possible to perform a set of inferences and correlations such that a group of possible scenarios that can be analyzed besides optimization parameters. Thus, the generalization provided for Bayesian networks is taken as an advantage for evaluating systems with strong correlation among their random variables.

\section{KDD, data mining and Bayesian networks}

The process of knowledge discovery in database (KDD) stands as a technology capable of widely cooperating in the search of existing knowledge in the data. Therefore, its main objective is to find valid and potentially useful patterns from the data.

The extraction of knowledge from data can be seen as a process with, at least, the following steps: understanding of the application domain, selection and preparation of the data, data mining, evaluation of the extracted knowledge and consolidation and the use of the extracted knowledge. Once in the data mining stage, considering the core of the KDD process, methods and algorithms are applied for the knowledge extraction from the database. This stage involves the creation of appropriate models representing patterns and relations identified in the data. The results of these models, after the evaluation by the analyst, specialist and/or final user are used to predict the values of attributes defined by the final user based on new data. In this work, the computational intelligence algorithm used for data mining was based on Bayesian networks.

A Bayesian network is composed of several nodes, where each node of the network represents a variable, that is, an attribute of the database; directed arcs connecting them implies in the relation of dependency that the variable can possess over the others; and finally probability tables for each node. 
The Bayesian networks can be seen as coding models of the probabilistic relationships between the variables that represent a given domain. These models possess as components a qualitative representation of the dependencies between the nodes and a quantitative (conditional probability tables of these nodes) structure, that can evaluate, in probabilistic terms, these dependencies. These components together provide an efficient representation of the joint probability distribution of the variables of a given domain.

One of the major advantages of the Bayesian networks is their semantics, which facilitates, given the inherent causal representation of these networks, the understanding and the decision making process for the users of these models. Basically, due to the fact that the relations between the variables of the domain can be visualized graphically, besides providing an inference mechanism that allows quantifying, in probabilistic terms, the effect of these relations [8].

\section{Scenario and Metrics}

The metrics are collected in a two-storey building made of bricks with rooms for Lectures, Computer and Telecommunication Labs and an anechoic camera, whose height occupies both the floors. The building has side glass windows with aluminum frames. The rooms are divided by walls built on bricks. There is a kitchen whose walls are covered with ceramic tiles. At the moment, the building is still unoccupied and with no furniture.

Fig. 1 shows the layouts of both the floors. The network access point under study is shown as a circle at the left side on the ground floor. The interference network, also represented as a circle, is located at the left side on the first floor. The distance that separates the two points is the height of the building.

\subsection{Testbed Features}

In the experiment, Compaq notebook under Windows $\mathrm{XP}{ }^{\odot}$ was associated with access point 802.11g Linksys ${ }^{\odot}$ WRT54G Router Speed Booster. This access point was connected to a $100 \mathrm{Mbps}$ Ethernet and one of the LAN ports was connected to the RADCOM ${ }^{\odot}$ protocol analyzer [9]. The other port of the protocol analyzer was connected to Toshiba Satellite notebook also under Windows XP $\odot$. The Compaq notebook was used to make VoIP calls to Toshiba notebook by using CallGen323 (program that makes 1-minute lasting calls) while Openphone [10] was used to receive calls. The metrics obtained in the application layer were monitored through packet loss, jitter, throughput and PMOS by using Performer Media Pro available within the protocol analyzer. A certain number of measurements were collected in an indoor environment. The same process was repeated by adding another network called interfering network. In this interfering network, 2 notebooks and another access point were used. Iperf software [11] was used to generate traffic within this net. 


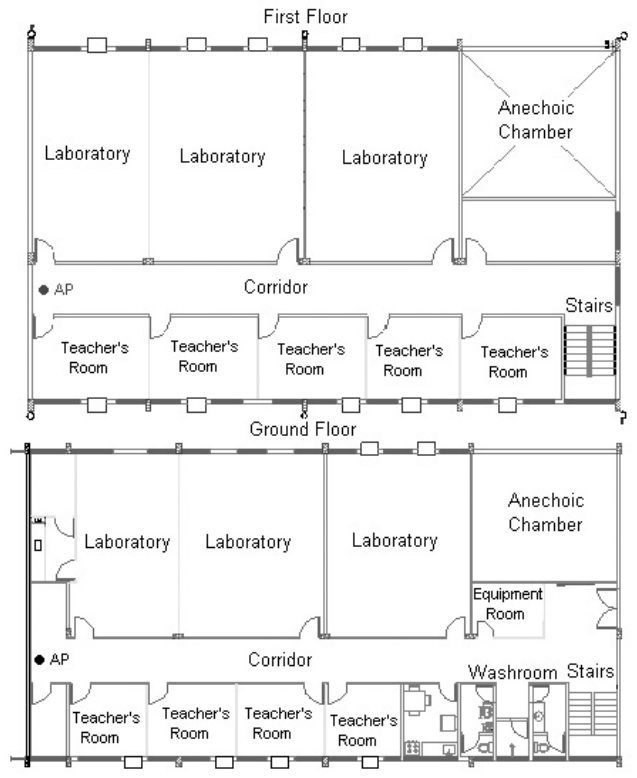

Fig. 1. The Layouts of ground and first floors.

\section{$5 \quad$ Methodology and Measurements}

In order to take the measurements, the locations were first selected and then were marked with an adhesive tape. Their distances from the walls were also measured. An access point was located at the entrance of the corridor situated in the ground floor. This network is called as network under study and it was programmed to use channel 7 (central frequency under $2.442 \mathrm{GHz}$ ). In this network, the AP is connected through a network cable to the protocol analyzer which in turn is connected to a receiving notebook. In order to generate VoIP calls, CallGen323, developed by Project OpenH323 [10], was used. The notebook that transmits the packets is positioned on a cart, that is moved to each point where the measurement is taken after remaining for 3 minutes at the previous location. The cart also carries another notebook to measure the power, received by wireless board from several access points, by using Network Stumbler ${ }^{\odot}$ software [12]. The same notebook was not utilized as the use of Network Stumbler ${ }^{\odot}$ does not allow the computer in which this software is running to be connected to a network.

During the measurements, the following parameters were stored: power (via software Netstumbler ${ }^{\odot}$ ), distance transmitter-receiver (obtained after treating data through the locations of the measuring points and access point), jitter, packet loss, PMOS and throughput (measured by protocol analyzer).

After this first phase of measurements, and new wireless network was installed called as interfering network using the same channel used by network under 
study. This new network was placed in the first floor pointing towards the same direction of the network under study Fig.1. Iperf [11] was the program used to generate the traffic as it allows to specify the time at which the traffic can be generated.

After this second phase, data were treated and the measurements were compared. The following section presents the results of this comparison.

\section{Some Inferences based on Bayesian Networks}

This section discusses the measurements of the application and physical layers as well as the results obtained by using Bayesian networks. The study involves treating the measured data with and without interference with the Bayesian network technique.

In any process of knowledge discovery, there is a pre-analysis phase of treatment (soft mining) of the data where information that is not going to contribute to the final result are removed. Hence, the input fields for the Bayesian network were obtained from the protocol analyzer after the pre-analysis. They worked as input to the free version Bayesware Discover ${ }^{\odot}$ (BDD) commercial software [13].

\subsection{Bayesian Networks without Inference}

When the Bayesian network is created to analyze the measurements with and without interfering network, each attribute will be turned into a network node as shown in Fig.2(a), Fig.2(b), Fig.3(a) and Fig.3(b).

In this work Bayesian networks were created representing the actual system that is based on the collected data. This network contains joint probability distribution tables of each node. It is based on dependencies, consisting of attributes i.e., possible values that each variable may assume and their respective probabilities. Once these networks are set and verified that they do represent the actual system and database, inferences are conducted on these Bayesian networks so that the verification of the WLAN network behavior as well as the results obtained from this inference is performed.

In Figures 3(a) and 3(b) both PMOS and packet loss are not related with any other measured parameter meaning that they do not vary, for instance, with the distance. This occurred during the measurement, when the network was busy just with one VoIP application which was not using the entire bandwidth. The relationship of QoS and physical layer for networks with and without interference can be identified graphically and it can be pointed that interference has a major impact in the probability relations as observed Fig.2(a), Fig.2(b), Fig.3(a) and Fig.3(b).

Fig.4 and Fig.5 capture the output window from running Bayesware Discoverer ${ }^{\odot}$ [13]. On the right side, the network is represented as a graph and the left side shows marginal probability distributions determined by Bayesian network, based on the measured data. Each of these probabilities represent Bayesian network's expectation of the WLAN nodes (jitter, PMOS, Packet-Loss, throughput represented as Band, D-Radio, power) [14]. 


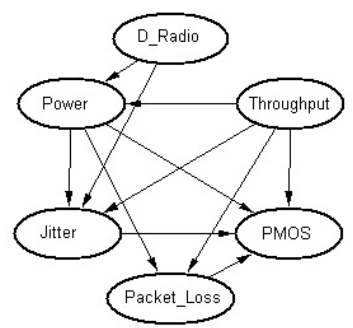

(a)

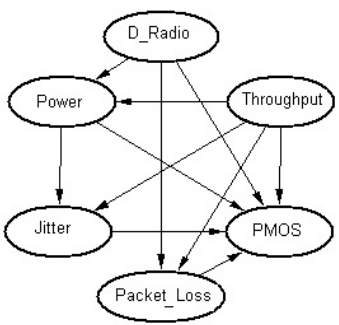

(b)

Fig. 2. Bayesian networks results(with interference) to: ground floor(a) and first floor(b).

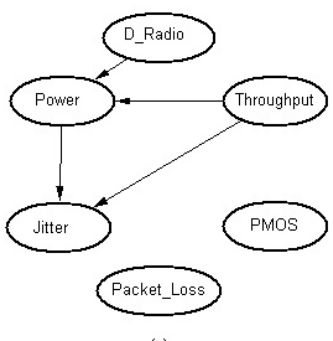

(a)

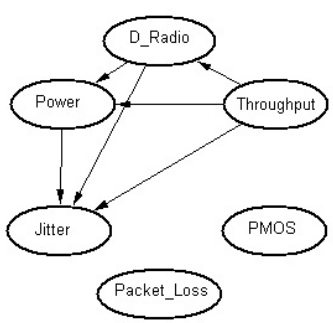

(b)

Fig. 3. Bayesian networks results(without interference) to: ground floor(a) and first floor(b).

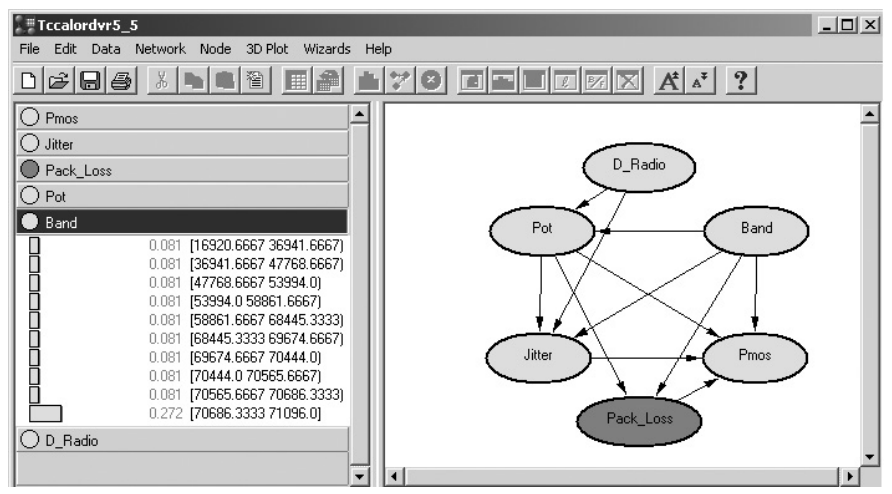

Fig. 4. Bayesian networks without inference with interference to ground floor.

\subsection{Bayesian Networks with Inference}

The most relevant results are shown in Fig.6, Fig.7, Fig.8 and Fig.9 for the two inferences that were conducted. The first inference refers to power with the largest value while the second refers to the worst throughput. 


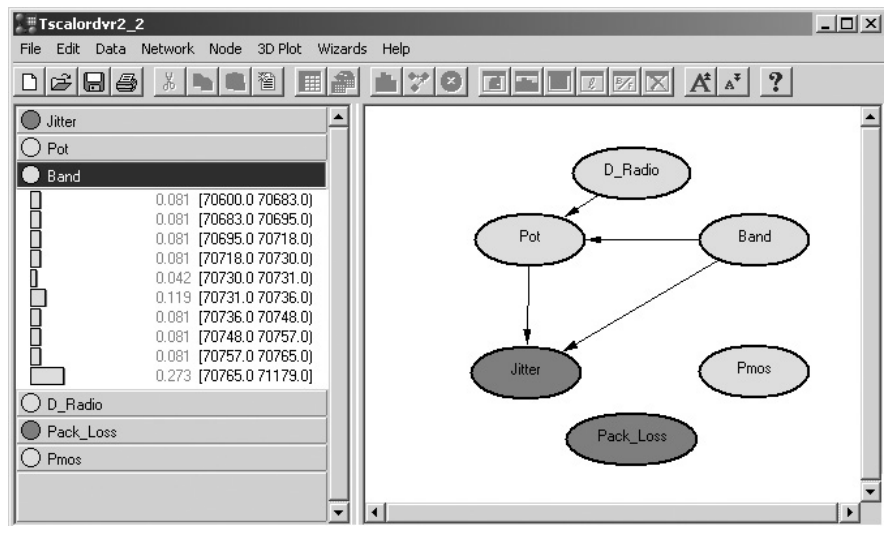

Fig. 5. Bayesian networks without inference without interference to ground floor.

One can observe (Fig.6 and Fig.7) that for the larger values assigned to power (ranging from -48.18 to $-41.81 \mathrm{dBm}$ ), in the first floor considering interference, the probability of throughput lying within 70686.33 and 71096.0 bps is $51.6 \%$. When interference is not considered, the probability of throughput lying within 707650.0 and $711790.0 \mathrm{bps}$ is $37.7 \%$. Therefore, there was a change in the network under study for this specific parameter, the throughput.

Similarly, results are also shown for other metrics. In the case of packet loss, the probability of no loss for network with interference is $55 \%$ whereas the value is $88 \%$ when interference is not considered. Considering now the jitter, its probability for lying within 4.33 to $6.66 \mathrm{~ms}$ is $32.7 \%$ for network with interference. This value increases to $84.6 \%$ for lying within 0 to $2 \mathrm{~ms}$ for network without interference. PMOS was also affected by the interference. Its probability values dropped down from $98.8 \%$ for lying within 3.34 to 4.0 (network without interference) to $49.3 \%$ for lying within 3.7 to 6.03 (network with interference). Finally, the distance metric had no significant variation as shown in Fig.6 and Fig.7.

Another evidence showing the influence of interference can be shown in the experiment in which the lowest throughput interval of 16920.66 to 36941.66 bps was selected. Fig.8 and Fig.9 show the results.

Bayesian networks that were generated (with and without interference) for the ground floor show that there is an effect from interference into the parameters as shown in Fig.8 and Fig.9. The inference value selected is different for these two situations (with and without interference).

The worst throughput for the network with interference lies within 16920,66 and 36941,66 bps whereas for the network without interference lies within 70600,0 a 70683,0 bps. This demonstrates that the throughput was reduced for the network with interference.

Now, moving to another parameter, the packet loss, the network with interference presented the largest probability (49.9\%) in the range $21 \%$ to $27 \%$. But 


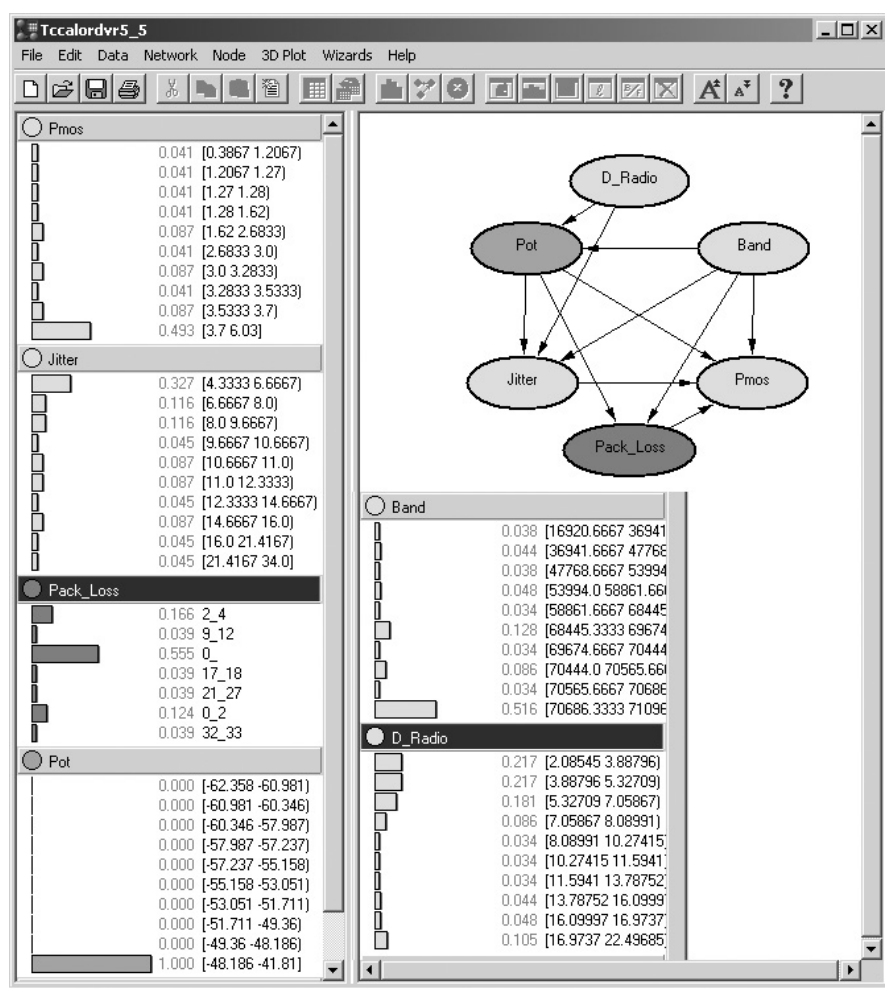

Fig. 6. Bayesian networks with largest power inference with interference applied to ground floor.

in the network without interference presented the largest probability $(88 \%)$ for the packet loss to be zero.

The probability of jitter to be above $9.66 \mathrm{~ms}$ has a probability of $78.3 \%$ in case of network with interference and the probability to be below $4 \mathrm{~ms}$ is $100 \%$ in case of network without interference.

PMOS, in network without interference, has its probability of $98.8 \%$ for lying within 3.34 and 4.0. The behavior of this metric in network with interference cannot be well characterized as it presented the same probability of $21 \%$ for both ranges of 0.38 to 1.2 and 3.7 and 6.03. However, it can be seen that there is an impact from the interference. Again, distance metric has not variation as shown in Fig. 8 and Fig.9. It is worth mentioning that the throughput values used in the inference of the worst throughput in networks with and without interference are different showing once more that interference, in fact, affects QoS parameters. 


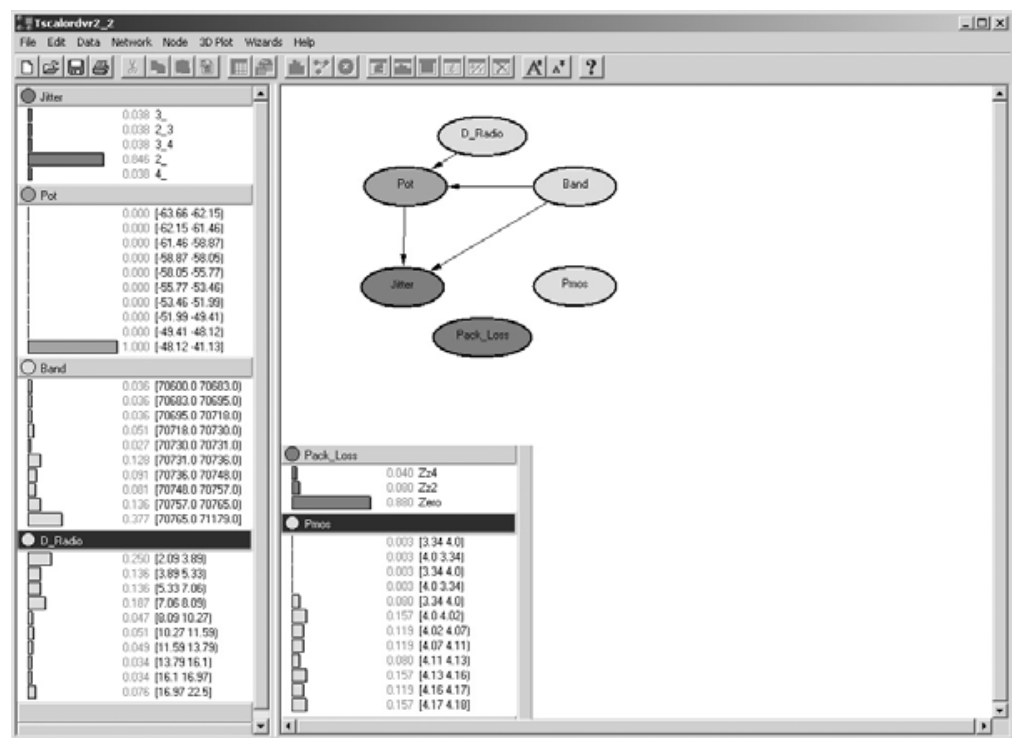

Fig. 7. Bayesian networks with largest power inference without interference applied to ground floor.

\section{Capacity Planning and Performance Evaluation Issues: A Methodology Proposal}

In real building there is a very strong trend to find similar scenarios to the presented ones in this paper, where different networks cohabit and where it is desirable that applications with rigid parameters of QoS carry out (e.g.: VoIP). To make this possible, the accomplishment of the following stages is necessary, at least, according to the methodology proposed in this paper:

- Characterization of the environment parameters (physical layer) of the WLAN: The presence of obstacles, like walls, environment aspects, among other, on the path of radio transmission attenuates signal power. In this way the reception quality is degraded and the error probability increases The power can vary significantly, since in a short periods, due to mobility and the multipath effect. Consequently retransmissions are produced and WLAN performance is influenced. It must be also considered the signal-to-noise ratio, often written $\mathrm{S} / \mathrm{N}$ or SNR, which from 10 decibels we can say that we have a suitable signal, from 10 to 15 decibels is enough, from 15 to 20 it is good signal and 20 in ahead is excellent. These numbers are recommendable for data; for voice 25 decibels or more are recommended and not to receive a signal from another access point that is working in the same channel and that is bigger than 10 decibels [5].

- Survey of the interfering networks: 


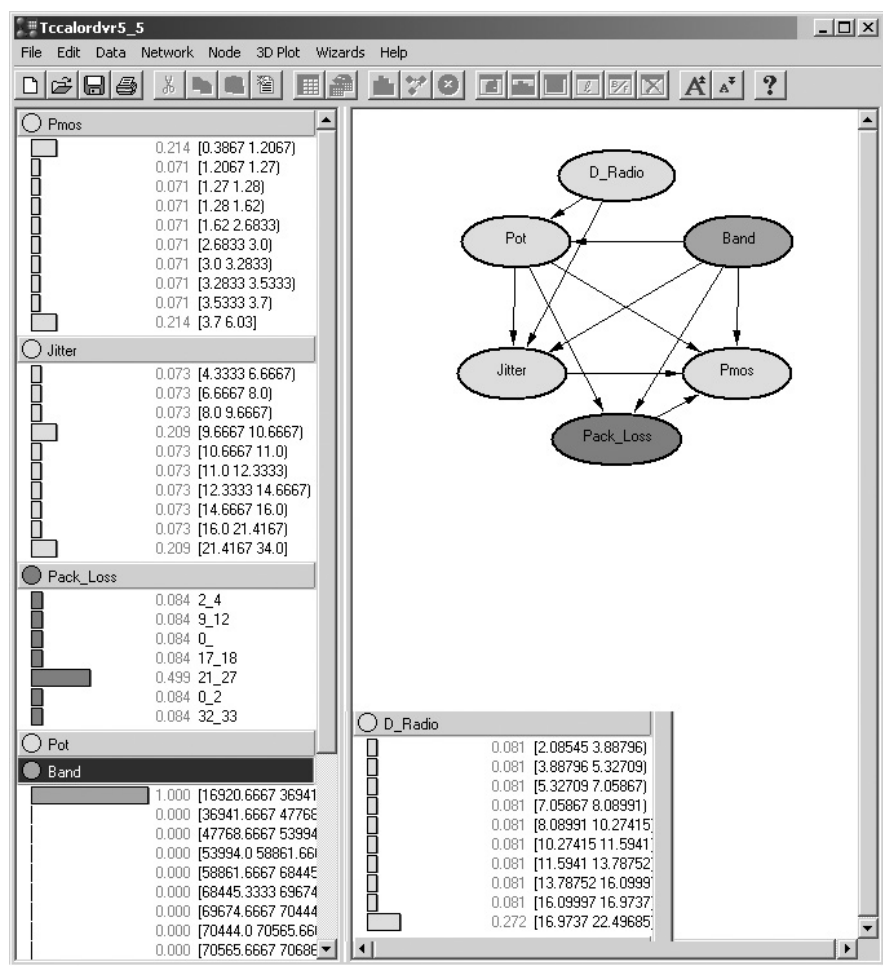

Fig. 8. Bayesian networks with worst throughput inference with interference applied to ground floor.

- Define the spatial distribution of the wireless network;

- Mark and to obtain the coordinates of access points to be evaluated;

- Start the application for wireless network to be studied;

- Use a software for collecting samples of signal level on wireless network;

- Use an application in the protocol analyzer to store QoS measures;

- Start application(s) in the interferent wireless network.

- Definition of QoS requirements of the target application:

There are several available metrics to measure the quality of a connection, such as packet loss rate, one-way delay, jitter, and throughput. Average oneway delay is probably the most critical parameter for VoIP. If it is too long, conversation flow is compromised and communication may become unnatural. ITU-T guidelines recommend a one-way delay of up to 150 milliseconds [15]. Beyond that, negative consequences gradually accrue. In IEEE 802.11 networks, the one-way delay between client and access point is usually less than 10 milliseconds, and therefore should not be a problem in VoIP. Jitter is the packet-to-packet variation in the one-way delay. Most modern systems will use some type of adaptive playback to smooth out the jitter, 


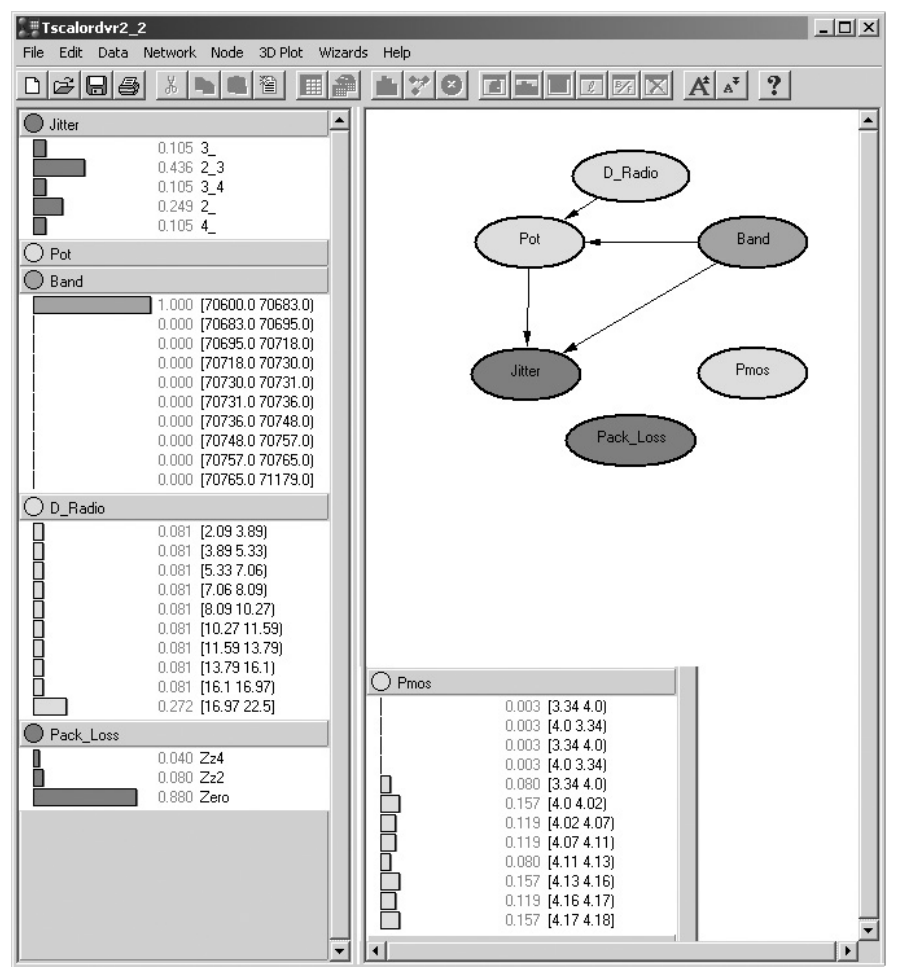

Fig. 9. Bayesian networks with worst throughput inference without interference applied to ground floor.

but this increases the one-way delay, and can introduce artifacts into the speech. In Wi-Fi networks, jitter is generally small, partially because oneway delay and packet sizes are small, too. However, at this paper showed that there are times when extreme delay variations can occur with significant impact on voice quality. Packet loss rate also affects speech quality, as the decoded speech will present artifacts associated with the lost packets. For VoIP, packet loss rates of up to $1 \%$ are generally acceptable [15]. In IEEE 802.11 networks, collisions and other losses are hidden by an automatic re-transmission strategy. Since these retransmissions are transparent to the application layer, the final packet loss rate is typically less than $1 \%$ and, therefore, acceptable for typical VoIP applications. Note, however, that as a mobile terminal gets out of the range, the loss rates increase abruptly; quickly making speech communication impossible. Throughput: the bandwidth required by a single VoIP connection is significantly less than the nominal capacity of IEEE 802.11 networks. Typical speech codecs require no more than $64 \mathrm{Kbps}$, while $802.11 \mathrm{~g}$ offers $54 \mathrm{Mbps}$. However, if the same access point is used to support multiple calls, we may have a capacity problem [15]. 
It is important to consider if there are WLANs near to our WLAN, where access points channels of our neighbors do not interfere with ours. Technologies that could produce interference are: Bluetooth, microwaves, some cellular phones and others WLANs, among others [5].

- Characterization of traffic for the target application:

- Running several iterations of the application target observing patterns and typical curves by means of acquisition of samples and utilization of goodness of fit tests;

- Definition of probability distribution for each performance measure studied, such as: delay, blocking probabilities and throughput;

- Definition of the measures that must be considered in the computational models (computational intelligence, optimization and simulation).

- Accomplishment of inferences based on simulation, computational intelligence or analytical models/optimization, to verify aspects as: correlation, possible scenarios, scalability, availability and performance.

\section{Conclusions}

This paper proposes the use of computational intelligence by employing Bayesian Networks to verify the interference influence in QoS parameters. Bayesian analysis showed this influence with precision, pointing out that interference has a major impact in the 802.11 network performance.

As showed in Section 2, the major difference in the work presented here from research cited in [3] and [7] lies in the field experiments with and without interference. A Bayesian network was used to analyze the data to show an evidence, as shown in [7], that there is an influence of the radio environment affecting QoS

parameters. However, the work presented in this paper includes investigation of other parameters such as jitter, packet loss and PMOS besides throughput that was the only parameter analyzed in [3] and [7]. The work hasn't included any explicit optimization study but some research is in progress in using Bayesian networks for this purpose.

The sequence of this work is to do new measurement campaign with multiple sources of traffic with and without interference and to draw the roadmap for capacity planning with an optimal number of APs, the cost function will be a Bayesian function and their weights and coefficients will be validated with the use of genetic algorithms and Markov chains for the scenery with and without interference, also proposed in [8] and validate the hypothesis suggested in [8] experimentally.

Only one user in the WLAN was used. One can extend Bayesian network to multiple user scenarios. It is important to mention that Bayesian network offers an approach to select several scenarios of QoS such that it is possible to guarantee a minimum distance to the AP for VoIP application in an indoor WLAN environment. 


\section{References}

1. Medepalli, K., Gopalakrishnan, P., Famolari, D., Kodama, T.: Voice Capacity of IEEE 80211b 80211a and 80211g Wireless LANs. Vehicular Telecommunications Conference (Globecomm 2004), IEEE Communications Society, Vol.3, 29Nov.-3Dec., pp. 1549-1553.

2. Zhai, H., Wang, J., Fang, Y.: Providing Statistical QoS Guarantee for Voice over IP in the IEEE 802.11 Wireless LANs. IEEE Wireless Communications, Vol. 3, Issue 1, Feb.(2006), pp. 36-43.

3. Lu, J. L., Jaffres-Runser, K., Gorce, J. M., Valois, F.: Indoor WLAN Planning with a QoS Constraint Based on a Markovian Performance Evaluation Model. IEEE International Conference on Wireless and Mobile Computing, Networking and Communications (WiMob'2006), June 19-21, pp. 152 - 158.

4. Glisic, S. G.: Advanced Wireless Networks: 4G Technologies. John Wiley and Sons LTDA, June 2006.

5. Diaz, L. E. N., Diaz, J. A. P.: A Model for Designing WLAN's 802.11 for VoIP. Proceedings of the Electronics. Electronics, Robotics and Automotive Mechanics Conference (CERMA'06), IEEE Computer Society, Vol. 2, Set.(2006), pp. 110-115.

6. Lu, J. L., Valois, F.: Performance evaluation of 802.11 WLAN in a real indoor environment. IEEE International Conference on Wireless and Mobile Computing, Networking and Communications(WiMob'2006), Montreal-Canada, June 19-21, pp. 140-147.

7. Garg, S., Kappes, M.: Can I add a VoIP call?. IEEE International Conference on Communications (ICC'03), Vol.2,11-15 May (2002), pp. 779-783.

8. Santana, A. L., et al.: Strategies for improving the modeling and interpretability of Bayesian Networks. Data Knowl. Eng. (2006), doi:10.1016/j.datak.2006.10.005

9. http://www.radcom.com/ acessed in 12/01/2006.

10. http://www.openh323.org/ acessed in 12/05/2006.

11. http://dast.nlanr.net/Projects/Iperf/ accessed in 12/19/2006.

12. http://www.netstumbler.com/ acessed in 12/19/2006.

13. Bayesware Discoverer,Copyright ${ }^{\odot} 2000$, Bayesware Limited. http://www.bayesware.com/, accessed in 01/05/07.

14. Bayesware Discoverer, User Manual, Copyright ${ }^{\odot}$ 2000, Bayesware Limited. http://www.bayesware.com/, accessed in 01/07/007.

15. Conceição, A., Li, J., Florêncio, D., Kon, F.:Is IEEE 802.11 ready for VoIP?. In 8th International Workshop on Multimedia Signal Processing (IEEE MMSP), Victoria, Canada, October 2006. 